single ionisation. The dotted curve refers to the observed masses and radii of planets and white dwarfs. The run of the theoretical curve is very similar to the observed curve. The theory thus explains the fundamental point that whereas for a white dwarf a smaller mass corresponds to a larger radius, for a planetary mass, which one can now say is only a black dwarf of mass less than about 1/1,000th the solar mass, a smaller mass corresponds to a smaller radius. The calculated radii of Jupiter and Saturn are about $3 \frac{1}{2}$ times too small. For other planets the agreement is better. However, we shall not enter into these details in this note.

We are attempting to refine the above theory in two ways : to evaluate the $\eta$ 's in (2), and to make the calculations for a mixture of elements. The presence of hydrogen, we find, while not appreciably modifying the theoretical curve in the region of lighter planets, raises it for heavier ones. The point we would like to emphasise here is that any satisfactory theory of the structure of stellar masses composed of degenerate matter must also combine with it the theory of ionisation in degenerate matter. This is what we have done, and we find that this combined theory is capable of explaining the broad features of planetary structure.

\footnotetext{
${ }^{1}$ Observatory, Sept. 1935, p. 260.
}

Milne, Mon. Not. Roy. Ast. Soc., 92, 610; 1932. In the above formula Milne's $\mu$ and $K$ have been replaced by $\mu m_{H}$ and $K / \mu^{5 / 3}$ respectively. (See, for example, Kothari, Mon. Not, Roy. Ast. Soc., 93, 74; 1932.)

' Kothari and Majumdar, Astro. Nach., 244, 74 ; 1931.

\title{
Population Trends
}

$\mathrm{I}^{\mathrm{T}}$ is now generally agreed that population problems lie near the root of many others-social, economic and political-and that there can be no constructive statecraft whilst nothing accurate is known of the quantity of a people. It is established that from the beginning of the nineteenth century until 1914, the population of England had increased by $1 \cdot 2$ per cent a year, that during the period 1914-24 this annual increase had fallen to 0.47 per cent, and that in 1924-34 further to 0.44 per cent. It is commonly agreed that the slowing down of a population increase in the second period was due to the high war mortality, to the decrease of immigration, and to the diminution of fertility; and that the smallness of the population increase in the third period was due to a further decrease in fertility and immigration. It is further accepted that the greatest reproductive rate of England and Wales is now less than unity, and that this implies that whatever changes in mortality ensue, a continuous decline of the total population is inevitable unless something happens which will increase fertility well above its present level. This being so, it is highly desirable that attempts should be made to project present trends in fertility and mortality into the immediate future.

This has been done very ingeniously by Dr. Fnid Charles in a Special Memorandum issued by the Executive Committee of the London and Cambridge Econornic Service*. In this, the author examines eertain questions and seeks to arrive at satisfactory answers. Assuming that fertility and mortality for each year of age continue to remain constant at the 1933 level, she arrives at the conclusion that an actual decline in the numbers of the population will ensue in 1943 ; that the population will be halved at the end of a century; that the school age group within the population will decrease to 79 per cent of the present figure twenty years from now, and will then constitute only $12 \cdot 6$ per cent of the total population; that the age group $60+$ will constitute $16 \cdot 6$ per cent of the total population; that the crude birth rate per thousand twenty-five years from now

* London and Cambridge Economic Service. Special Memorandum No. 40: The Effect of Present Trends in Fertility and Mortality upon position. Pp. 20. (London: C/o London School of Economics, 1935.) posit. will be approximately 12 , and the crude death rate 16.5 ; fifty years hence the crude birth rate will be 11 , and the crude death rate $20 \cdot 5$; and finally, that the percentage age composition of the population will begin to oscillate about a stable level after about sixty years.

Assuming, on the other hand, that fertility and mortality continue to fall in the manner suggested by the figures available for the last decade, it seems probable that an actual decline in numbers in the population will ensue in 1939 ; that the population will be reduced to one-tenth at the end of a century; that the school age group will decrease to 57 per cent twenty years from now ; and that this group will constitute 9.5 per cent of the total population, whereas the $60+$ age group will constitute 18.6 per cent. The crude birth rate twenty-five years from now will be 6 per cent, and the erude death rate 14 per cent. Fifty years hence, the crude birth rato will be 2.5 per cent and the crude death rate 24 per cent.

Considering a third assumption, that mortality continues to fall and fertility remains constant at the 1931 level (and Dr. Charles argues that this, together with the first assumption, are conservative estimates of the immediate prospect of declining population, whilst the second represents a more reasonable forecast of the trend of population), she shows that the actual decline in numbers of the population will be felt between 1960 and 1965 .

The effect of this declining fertility will be to raise the sex ratio of the total population owing to the preponderance of females in the older age groups. Between the ages of twenty and fifty years the present ratio of females to males is $1 \cdot 099$. This would appear to be abnormally disturbed on account of the loss of male lives during the Great War; for, according to the arguments based on any one of the three assumptions, there are fewer females than males in this age group in 1965 and hereafter.

This paper is an outstanding contribution to knowledge; it represents probably the most im. portant use to which census figures have been put for many a year. 